\title{
Motivational Factors And The Hospitality Industry: A Case Study Examining The Effects Of Changes In The Working Environment
}

Dr. Thomas Marshall, Les Roches Gruyère University of Applied Sciences, Switzerland Dr. Ewa M. Mottier, Les Roches Gruyère University of Applied Sciences, Switzerland Dr. Robert A. Lewis, Les Roches Gruyère University of Applied Sciences, Switzerland

\begin{abstract}
The Mandarin Oriental Hotel in Bangkok is a world-wide leader in hospitality management. A critical ingredient for their success has been developing and maintaining superior performance from their employees. How is that accomplished? What Human Resource Management (HRM) practices should organizations invest in to acquire and retain great employees? Recent studies have demonstrated the importance of providing a supportive work environment, one that engenders employee satisfaction and promotes effective service performance among hospitality employees. But what is a supportive environment for hotel workers who are required to be responsive to the needs of guests twenty four hours a day? The Mandarin Oriental's unique approach involved building a separate area in the hotel dedicated to the well-being of their employees. This area-The O-Zone-originated from the idea of providing workplaces which support fun and enjoyment as part of work-life balance.
\end{abstract}

The case focuses attention on how changes in the working environment can significantly enhance the recognition and appreciation of employees $A$ dedicated employee center in the hospitality industry has not been explored before and offers up an exceptional opportunity to examine the effect on motivation and how the center may be appreciated differently by employees based on gender, department and work experience-an important consideration for Human Resources when designing incentive programs.

Keywords: Hotel Work Environment; Management Commitment; Employee Motivation; Mandarin Oriental

\section{LEARNING OBJECTIVES:}

1. To evaluate intrinsic motivation factors for employees in a Thai luxury hotel.

2. To identify extrinsic motivation factors for employees in a Thai luxury hotel.

3. To gain insight into employee motivation in a Thai luxury hotel context.

\section{CASE SYNOPSIS}

\subsection{A "hotel within a hotel" at the Mandarin Oriental Bangkok: The O-Zone experience}

The O-Zone was conceived as an experiment placing the Mandarin Oriental Bangkok at the forefront of the hospitality industry by providing superior working conditions for their employees. The idea originated from workplaces that emphasized fun and enjoyment as part of work-life balance. It is interesting to note that these workplaces have traditionally been in the non-service sector, which also in many cases is 'innovative' by nature. By contrast, the service sector, and more specifically hotels, despite its use of selected innovative practices, has traditionally not extended these practices to address basic employee needs, such as working conditions. For over 135 years Mandarin Oriental Bangkok has been a pioneer in providing a unique guest environment, such as being the first hotel in Bangkok to have running water or elevators (Lewis and Mottier, 2011). Continuing this tradition, the 
O-Zone has attempted to offer exceptional working conditions, incorporating fun and centralizing employee requirements into one unique center.

\section{BACKGROUND}

Pfeffer (1994) emphasizes that in order to succeed in a global business environment organizations must invest in HRM sufficiently to acquire and retain employees who possess better skills and capabilities than their competitors. This investment will be their competitive advantage. Despite this recognition of the importance of employee development, the hospitality industry has historically been dominated by underdeveloped HR practices (Lucas, 2002). This study focused on the development of one practice, illustrated by an employee-dedicated center within a luxury hotel.

Lucas also points out that "the substance of HRM practices does not appear to be designed to foster constructive relations with employees or to represent a managerial approach that enables developing and drawing out the full potential of people, even though employees may be broadly satisfied with many aspects of their work" (Lucas, 2002). In addition, or maybe as a result, high employee turnover has been a recurring problem throughout the industry. Among the many cited reasons are low compensation, inadequate benefits, poor working conditions and compromised employee morale and attitudes (Maroudas et al., 2008). These studies reveal the need to investigate these factors empirically.

$\mathrm{Ng}$ and Sorensen (2008) demonstrated that when managers provide recognition to employees, motivate employees to work together, and remove obstacles preventing effective performance, employees feel more obligated to stay with the company. This was succinctly summarized by Michel et al. (2013): "[P]roviding support to employees gives them the confidence to perform their jobs better and the motivation to stay with the organization." Hospitality organizations can therefore enhance employee motivation and retention through the development and improvement of their working conditions. These conditions are inherently linked to the working environment.

While it seems likely that employees' reactions to their job characteristics could be affected by a predisposition to view their work environment negatively, no evidence exists to support this hypothesis (Spector et al., 2000). However, self-enhancing bias exists (e.g., Ferrell \& Weaver, 1978; Kantor \& Weisberg, 2002), and is likely to have skewed data in favor of the respondents. It is also recognized that, given the opportunity, many people will find something to complain about in relation to their workplace (Poulston, 2009). There is therefore a strong link between the perceptions of employees and the recognizable hygiene factors of their work environment.

Such conditions are particularly troubling for the luxury hotel market, where high-quality service, requiring a sophisticated approach to HRM, is recognized as a critical source of competitive advantage. (Maroudas et al., 2008) In a real sense, the services of hotel employees represent their industry (Schneider and Bowen, 1993). This representation has commonly been limited to guest experiences. This suggests that there has been a dichotomy between the guest environment provided in luxury hotels and the working conditions of their employees.

It is therefore essential for hotel management to develop HRM practices that enable them to inspire and retain competent employees. This requires an understanding of what motivates employees at different levels of management and different stages of their careers (Enz and Siguaw, 2000). This implies that it is beneficial for hotel managers to understand what practices are most favorable to increase employee satisfaction and retention.

Herzberg's (1966) motivation-hygiene theory proposes that people have two major types of needs, the first being hygiene needs which relate to the context in which work is performed. Among these needs are working conditions or extrinsic motivation factors. When these factors are unfavorable, job dissatisfaction may result. Significantly, though, just fulfilling hygiene needs does not result in satisfaction, but only in the reduction of dissatisfaction (Maroudas et al., 2008). Taking Herzberg's (1966) theory into the context of a luxury hotel, the recognition of employees' hygiene needs can positively affect job satisfaction.

Employees also have intrinsic motivation needs or motivators, which include such factors as achievement and recognition. Compared to hygiene factors, which result in a neutral satisfaction state, motivator factors may 
ideally result in job satisfaction (Maroudas et al., 2008). Again, Herzberg's (1966) theory discusses the need for a "balance" of these two types of needs.

The impact of fun as a motivating factor at work has also been recently explored. Building on earlier work of Peluchette and Karl (2005) whereby fun experienced on the job was positively related to job satisfaction for healthcare employees, Tews, Michel and Stafford (2013) conducted a study focusing on waitpersons from a casualthemed restaurant chain in the United States. It was found that fun activities had a favorable impact on performance and manager support for fun had a favorable impact in reducing turnover. Their findings support that fun may indeed have a beneficial effect, but the framing of that fun must be carefully aligned with both organizational goals and employee characteristics. "Manager must learn how to achieve the delicate balance of allowing employees the freedom to enjoy themselves at work while simultaneously maintain high levels of performance" (Tews et al., 2013). The previous arguments which discuss the importance of job satisfaction factors in hotels suggest that it is beneficial to investigate the role of fun in the hospitality context.

Additionally, Simons and Enz (1995) propose that employees from different departments respond in different ways to job rewards. This suggests that individual differences should be considered when designing incentive programs. Using data from six multinational luxury hotel chains in Athens, Maroudas et al. (2008) categorized such differences to include work experience, gender and age when considering organizational incentives. Following on that research, it is the contention of this paper that these differences may also hold true when designing an employee well-being center, incorporating both extrinsic and intrinsic factors.

Rowley and Purcell (2001) investigated the role of potential causes of turnover such as stress and burnout as well as false expectations and poor management practices. Margaret Deery (2008) has recommended several actions that can be adopted at the organizational level to retain good staff as well as assist in balancing work and family life. Specifically related to the implementation of O-Zone at Mandarin Oriental are:

- $\quad$ Allowing adequate breaks during the working day

- $\quad$ Staff functions that involve families

- $\quad$ Providing health and well-being opportunities

This research builds on the employee motivation measurement methodology of Maroudas et al. (2008) and follows previous motivation and satisfaction studies involving Thai hotel employees. Kim et al. (2009) investigated the effects of management commitment to factors affecting employees' job satisfaction and service behaviors at ten hotels in Bangkok. Their structural equation model indicated that rewards, empowerment and training are positively related to job satisfaction. Furthermore, job satisfaction was found to have a significant influence on Thai employees' customer service behaviors and cooperation. Job satisfaction may therefore serve as a mediator between HRM initiatives (here examined as a dedicated employee center) and employees' service behaviors toward customers and co-workers.

\section{METHODOLOGY}

The study took place at the Mandarin Oriental Bangkok, a multinational luxury hotel which seeks to differentiate through high-quality services and an HRM strategy of strong customer orientation. A questionnaire was developed to determine employee reaction to facility changes and their effect on employee motivation. The participants (161 respondents: 91 male and 70 female) voluntarily answered questionnaires in English and Thai. Each participant completed the questionnaire only once, and chose the language they were most comfortable with. These questionnaires contained ranked statements which could be responded to using a standard five-point scale.

Ten noteworthy facility changes were incorporated into the new employee relaxation area, the O-Zone:

1. Wall of Fame-recognizes employees with singular accomplishments

2. Fashion Zone - offers employee uniform cleaning and maintenance

3. His and Her - a personal grooming area

4. Health Zone — availability of a nurse or doctor for employees' healthcare concerns 
5. Café 48 - provides snacks and refreshments at a discounted price

6. Kiosk 48-sells Mandarin Oriental items at an employee discount

7. Colleagues Concierge - a specialized service for employee needs

8. Mind Zone - a dedicated area for reading and concentration

9. Dream Zone - a dedicated area for relaxation and meditation

10. Relax Zone - a fun area where employees can be themselves

This study builds on research into employee motivation in the luxury hotel industry and attempts to detect any significance among gender, department and work experience in relation to changes in hygiene factors (facilities) created by the new O-Zone.

\section{RESULTS AND DISCUSSION}

Study participants illustrated how intrinsic and extrinsic needs were met through their experiences in the OZone.

\subsection{Motivation}

Specific citations from employees indicating that intrinsic motivation needs had been addressed:

- "Sometimes it is not only salary, it is the thought that counts."

- $\quad$ "Because of the good environment, it helps me enjoy working more."

\subsection{Work Environment}

Likewise, the O-Zone's facilities had a positive effect on well-being in terms of addressing the extrinsic factors affecting hygiene needs of the work environment.

Specific citations from employees indicating that extrinsic hygiene factors had been addressed:

- "With a very pleasant working environment (with the O-Zone), it really makes me want to work enthusiastically."

- "This is a truly happy workplace which makes us different from other hotels."

- "The O-Zone improves harmony between colleagues."

Shifting from a qualitative impression to a more descriptive presentation of facilities, the following table provides participants' scaled responses to the O-Zone questionnaire. 
Table 1. Likert scale* findings in term of perceptions of employee facilities after the implementation of the O-Zone

\begin{tabular}{|c|c|c|c|c|c|}
\hline $\begin{array}{c}\text { Opinion of } \\
\text { O-Zone Facilities }\end{array}$ & Mean & Mode & $\begin{array}{c}\text { Skewness } \\
\text { Statistic }\end{array}$ & $\begin{array}{c}\text { Std. Error of } \\
\text { Skewness }\end{array}$ & $\begin{array}{c}\text { Cronbach's } \\
\text { Alpha Coefficient }\end{array}$ \\
\hline & & & & & $\begin{array}{c}0.802^{1} \\
(0.811)^{2}\end{array}$ \\
\hline Wall of Fame' & 4.10 & 4.00 & -1.589 & .190 & $0.794^{3}$ \\
\hline Fashion Zone & 3.98 & 4.00 & -1.187 & .190 & 0.802 \\
\hline His and Her & 4.06 & 4.00 & -2.090 & .190 & 0.789 \\
\hline Health Zone & 4.18 & 4.00 & -1.470 & .190 & 0.791 \\
\hline Café 48 & 4.46 & 5.00 & -2.273 & .190 & 0.787 \\
\hline Kiosk 48 & 4.38 & 5.00 & -1.771 & .190 & 0.785 \\
\hline Colleagues Concierge & 3.91 & 4.00 & -1.928 & .190 & 0.784 \\
\hline Mind Zone & 4.12 & 4.00 & -2.177 & .190 & 0.801 \\
\hline Dream Zone & 3.32 & 4.00 & -1.169 & .190 & 0.791 \\
\hline Relax Zone & & & & & 0.768 \\
\hline Game area & 3.89 & 4.00 & -.975 & .190 & 0.778 \\
\hline Reading corner & 4.02 & 4.00 & -1.162 & .190 & 0.785 \\
\hline \multirow[t]{2}{*}{ Internet corner } & 3.98 & 4.00 & -1.372 & .190 & 0.794 \\
\hline & 4.03 & 4.00 & & & \\
\hline
\end{tabular}

${ }^{\mathrm{T}}$ Summated scale Cronbach coefficient, which indicates internal consistency reliability

${ }^{2}$ Cronbach's Alpha based on standardized items

${ }^{3}$ Cronbach's Alpha if item deleted

*Based on a five-point Likert scale ranging from negative to positive

The following table examines the first of our possible relationships: Facilities and Gender.

Table 2. Cross-tabulation in term of perceptions of employee facilities after the implementation of the O-Zone as a function of gender

\begin{tabular}{|c|c|c|c|c|c|}
\hline $\begin{array}{c}\text { Opinion of } \\
\text { O-Zone Facilities } \\
\end{array}$ & Gender & Negative* & Positive & $\begin{array}{c}\text { Very } \\
\text { Positive* }\end{array}$ & $X^{2}$ and $p$-value \\
\hline Wall of Fame' & $\begin{array}{l}\text { Male } \\
\text { Female }\end{array}$ & $\begin{array}{l}16.5 \% \\
15.7 \% \\
\end{array}$ & $\begin{array}{l}56.0 \% \\
48.6 \%\end{array}$ & $\begin{array}{l}27.5 \% \\
35.7 \%\end{array}$ & $X^{2}=1.298, p=0.522$ \\
\hline Fashion Zone & $\begin{array}{l}\text { Male } \\
\text { Female }\end{array}$ & $\begin{array}{l}19.8 \% \\
21.1 \% \\
\end{array}$ & $\begin{array}{l}54.9 \% \\
60.6 \% \\
\end{array}$ & $\begin{array}{l}25.3 \% \\
18.3 \% \\
\end{array}$ & $X^{2}=1.125, p=0.570$ \\
\hline His and Her & $\begin{array}{l}\text { Male } \\
\text { Female }\end{array}$ & $\begin{array}{l}11.5 \% \\
11.1 \% \\
\end{array}$ & $\begin{array}{l}57.5 \% \\
55.6 \% \\
\end{array}$ & $\begin{array}{l}31.0 \% \\
33.3 \% \\
\end{array}$ & $X^{2}=0.096, p=0.953$ \\
\hline Health Zone & $\begin{array}{l}\text { Male } \\
\text { Female }\end{array}$ & $\begin{array}{l}11.2 \% \\
15.3 \% \\
\end{array}$ & $\begin{array}{l}54.4 \% \\
51.4 \% \\
\end{array}$ & $\begin{array}{l}34.4 \% \\
33.3 \% \\
\end{array}$ & $X^{2}=0.621, p=0.733$ \\
\hline Café 48 & $\begin{array}{l}\text { Male } \\
\text { Female }\end{array}$ & $\begin{array}{l}3.3 \% \\
0.0 \% \\
\end{array}$ & $\begin{array}{l}44.0 \% \\
50.7 \%\end{array}$ & $\begin{array}{l}52.7 \% \\
49.3 \% \\
\end{array}$ & $X^{2}=2.821, p=0.244$ \\
\hline Kiosk 48 & $\begin{array}{l}\text { Male } \\
\text { Female }\end{array}$ & $\begin{array}{l}6.6 \% \\
9.8 \% \\
\end{array}$ & $\begin{array}{l}42.9 \% \\
42.3 \% \\
\end{array}$ & $\begin{array}{l}50.5 \% \\
47.9 \% \\
\end{array}$ & $X^{2}=0.591, p=0.744$ \\
\hline Colleagues Concierge & $\begin{array}{l}\text { Male } \\
\text { Female }\end{array}$ & $\begin{array}{l}17.3 \% \\
17.4 \% \\
\end{array}$ & $\begin{array}{l}56.3 \% \\
50.7 \% \\
\end{array}$ & $\begin{array}{l}26.4 \% \\
31.9 \% \\
\end{array}$ & $X^{2}=0.620, p=0.733$ \\
\hline Mind Zone & $\begin{array}{l}\text { Male } \\
\text { Female }\end{array}$ & $\begin{array}{l}11.5 \% \\
12.7 \% \\
\end{array}$ & $\begin{array}{l}51.7 \% \\
49.3 \% \\
\end{array}$ & $\begin{array}{l}36.8 \% \\
38.0 \% \\
\end{array}$ & $X^{2}=0.107, p=0.948$ \\
\hline Dream Zone & $\begin{array}{l}\text { Male } \\
\text { Female }\end{array}$ & $\begin{array}{l}32.9 \% \\
52.3 \% \\
\end{array}$ & $\begin{array}{l}48.8 \% \\
34.3 \% \\
\end{array}$ & $\begin{array}{l}18.3 \% \\
13.4 \% \\
\end{array}$ & $X^{2}=5.667, p=0.059$ \\
\hline Relax Zone & & & & & \\
\hline Game area & $\begin{array}{l}\text { Male } \\
\text { Female }\end{array}$ & $\begin{array}{l}24.2 \% \\
32.4 \% \\
\end{array}$ & $\begin{array}{l}52.7 \% \\
39.4 \% \\
\end{array}$ & $\begin{array}{l}23.1 \% \\
28.2 \%\end{array}$ & $X^{2}=2.885, p=0.236$ \\
\hline Reading corner & $\begin{array}{l}\text { Male } \\
\text { Female }\end{array}$ & $\begin{array}{l}20.9 \% \\
15.5 \% \\
\end{array}$ & $\begin{array}{l}54.9 \% \\
54.9 \% \\
\end{array}$ & $\begin{array}{l}24.2 \% \\
29.6 \% \\
\end{array}$ & $X^{2}=1.063, p=0.588$ \\
\hline Internet corner & $\begin{array}{l}\text { Male } \\
\text { Female }\end{array}$ & $\begin{array}{l}24.1 \% \\
24.6 \% \\
\end{array}$ & $\begin{array}{l}40.7 \% \\
47.8 \% \\
\end{array}$ & $\begin{array}{l}35.2 \% \\
27.5 \% \\
\end{array}$ & $X^{2}=1.181, p=0.554$ \\
\hline
\end{tabular}


It was interesting to note there were no differences in terms of gender in regard to perception of the new facilities. This is in contrast to recent studies (Gherardi, 1996; Maroudas et al., 2008) where males have shown a tendency for an enhanced need and practice of socialization which would presumably be facilitated by the new social facilities.

The next table examines the second of tested relationships: Facilities and Departments.

Table 3. Cross-tabulation in term of perceptions of employee facilities after the implementation of the O-Zone as a function of department

\begin{tabular}{|c|c|c|c|c|c|}
\hline $\begin{array}{c}\text { Opinion of } \\
\text { O-Zone Facilities } \\
\end{array}$ & Department & Negative & Positive & Very Positive & $X^{2}$ and p-value \\
\hline \multirow{4}{*}{ Wall of Fame' } & $M g t \& A d m i n$ & $16.4 \%$ & $47.5 \%$ & $36.1 \%$ & \multirow{4}{*}{$X^{2}=7.850, p=0.249$} \\
\hline & Front Office & $25.0 \%$ & $35.0 \%$ & $40.0 \%$ & \\
\hline & Housekeeping & $0.0 \%$ & $58.3 \%$ & $41.7 \%$ & \\
\hline & Food \& Beverage & $16.2 \%$ & $60.3 \%$ & $23.5 \%$ & \\
\hline \multirow[t]{4}{*}{ Fashion Zone } & $M g t \& A d m i n$ & $24.6 \%$ & $50.8 \%$ & $24.6 \%$ & \multirow{4}{*}{$X^{2}=5.881, p=0.437$} \\
\hline & Front Office & $20.0 \%$ & $75.0 \%$ & $5.0 \%$ & \\
\hline & Housekeeping & $15.4 \%$ & $53.8 \%$ & $30.8 \%$ & \\
\hline & Food \& Beverage & $17.6 \%$ & $57.4 \%$ & $25.0 \%$ & \\
\hline \multirow[t]{4}{*}{ His and Her } & $M g t \& A d \min$ & $11.5 \%$ & $54.1 \%$ & $34.4 \%$ & \multirow{4}{*}{$X^{2}=6.545, p=0.365$} \\
\hline & Front Office & $21.1 \%$ & $68.4 \%$ & $10.5 \%$ & \\
\hline & Housekeeping & $7.6 \%$ & $46.2 \%$ & $46.2 \%$ & \\
\hline & Food \& Beverage & $9.1 \%$ & $56.1 \%$ & $34.8 \%$ & \\
\hline \multirow[t]{4}{*}{ Health Zone } & Mgt\&Admin & $14.8 \%$ & $45.9 \%$ & $39.3 \%$ & \multirow{4}{*}{$X^{2}=13.854, p=0.031 *$} \\
\hline & Front Office & $20.0 \%$ & $75.0 \%$ & $5.0 \%$ & \\
\hline & Housekeeping & $0.0 \%$ & $38.5 \%$ & $61.5 \%$ & \\
\hline & Food \& Beverage & $11.8 \%$ & $54.4 \%$ & $33.8 \%$ & \\
\hline \multirow[t]{4}{*}{ Café 48} & Mgt\&Admin & $1.6 \%$ & $41.0 \%$ & $57.4 \%$ & \multirow{4}{*}{$X^{2}=6.890, p=0.331$} \\
\hline & Front Office & $0.0 \%$ & $57.9 \%$ & $42.1 \%$ & \\
\hline & Housekeeping & $0.0 \%$ & $23.1 \%$ & $76.9 \%$ & \\
\hline & Food \& Beverage & $2.9 \%$ & $52.2 \%$ & $44.9 \%$ & \\
\hline \multirow[t]{4}{*}{ Kiosk 48} & $M g t \& A d \min$ & $6.5 \%$ & $41.0 \%$ & $52.5 \%$ & \multirow{4}{*}{$X^{2}=11.928, p=0.064$} \\
\hline & Front Office & $25.0 \%$ & $30.0 \%$ & $45.0 \%$ & \\
\hline & Housekeeping & $8.3 \%$ & $25.0 \%$ & $66.7 \%$ & \\
\hline & Food \& Beverage & $4.3 \%$ & $49.3 \%$ & $46.4 \%$ & \\
\hline \multirow[t]{4}{*}{ Colleagues Concierge } & Mgt\&Admin & $13.1 \%$ & $62.3 \%$ & $24.6 \%$ & \multirow{4}{*}{$X^{2}=25.181, p=0.000^{*}$} \\
\hline & Front Office & $52.7 \%$ & $36.8 \%$ & $10.5 \%$ & \\
\hline & Housekeeping & $10.0 \%$ & $30.0 \%$ & $60.0 \%$ & \\
\hline & Food \& Beverage & $12.2 \%$ & $53.0 \%$ & $34.8 \%$ & \\
\hline \multirow[t]{4}{*}{ Mind Zone } & Mgt\&Admin & $8.2 \%$ & $52.5 \%$ & $39.3 \%$ & \multirow{4}{*}{$X^{2}=13.134, p=0.041 *$} \\
\hline & Front Office & $5.0 \%$ & $75.0 \%$ & $20.0 \%$ & \\
\hline & Housekeeping & $0.0 \%$ & $46.2 \%$ & $53.8 \%$ & \\
\hline & Food \& Beverage & $20.3 \%$ & $40.6 \%$ & $39.1 \%$ & \\
\hline \multirow[t]{4}{*}{ Dream Zone } & Mgt\&Admin & $40.7 \%$ & $44.1 \%$ & $15.3 \%$ & \multirow{4}{*}{$X^{2}=7.491, p=0.278$} \\
\hline & Front Office & $52.6 \%$ & $42.1 \%$ & $5.3 \%$ & \\
\hline & Housekeeping & $33.4 \%$ & $22.2 \%$ & $44.4 \%$ & \\
\hline & Food \& Beverage & $42.0 \%$ & $41.9 \%$ & $16.1 \%$ & \\
\hline \multicolumn{6}{|l|}{ Relax Zone } \\
\hline \multirow[t]{4}{*}{ Game area } & Mgt\&Admin & $26.2 \%$ & $50.8 \%$ & $23.0 \%$ & \multirow{4}{*}{$X^{2}=14.792, p=0.022 *$} \\
\hline & Front Office & $60.0 \%$ & $30.0 \%$ & $10.0 \%$ & \\
\hline & Housekeeping & $25.0 \%$ & $33.3 \%$ & $41.7 \%$ & \\
\hline & Food \& Beverage & $20.3 \%$ & $49.3 \%$ & $30.4 \%$ & \\
\hline Reading corner & $M g t \& A d \min$ & $21.3 \%$ & $55.7 \%$ & $23.0 \%$ & \\
\hline & Front Office & $25.0 \%$ & $65.0 \%$ & $10.0 \%$ & $x^{2}=7023 n=0244$ \\
\hline & Housekeeping & $8.3 \%$ & $41.7 \%$ & $50.0 \%$ & $X=7.923, p=0.244$ \\
\hline & Food \& Beverage & $15.9 \%$ & $52.2 \%$ & $31.9 \%$ & \\
\hline Internet corner & Mgt\&Admin & $26.2 \%$ & $32.8 \%$ & $41.0 \%$ & \\
\hline & Front Office & $45.0 \%$ & $40.0 \%$ & $15.0 \%$ & \\
\hline & Housekeeping & $16.7 \%$ & $50.0 \%$ & $33.3 \%$ & $X^{-}=11.379, p=0.077$ \\
\hline & Food \& Beverage & $17.9 \%$ & $52.2 \%$ & $29.9 \%$ & \\
\hline
\end{tabular}


Four new facilities in O-Zone showed significant differences in employee perception. These facilities were: the Health Zone, where employees could visit the hotel nurse or doctor; the Colleagues Concierge, a service where employees could ask for transportation information or other miscellaneous requests from a dedicated team of three employees; the Mind Zone, an area dedicated to work or study; and the game area in the Relax Zone. These results may present an insightful corollary to Simons and Enz (1995). Now not only do employees from different departments respond in different ways to job rewards offered by the company, but they also respond differently to changes in dedicated employee facilities. Additionally, it is interesting to note that in all four significantly different facility changes, Housekeeping had the most positive perception.

The next table examines the third tested relationship: Facilities and Work Experience

Table 4. Cross-tabulation in term of perceptions of employee facilities after the implementation of the O-Zone as a function of length of work

\begin{tabular}{|c|c|c|c|c|c|}
\hline $\begin{array}{c}\text { Opinion of } \\
\text { O-Zone facilities }\end{array}$ & $\begin{array}{c}\text { Length of } \\
\text { work (years) }\end{array}$ & Negative & Positive & Very Positive & $X^{2}$ and p-value \\
\hline \multirow[t]{3}{*}{ Wall of Fame' } & $<2$ & $11.5 \%$ & $42.3 \%$ & $46.2 \%$ & \multirow{3}{*}{$X^{2}=5.489, p=0.241$} \\
\hline & $2-5$ & $20.0 \%$ & $60.0 \%$ & $20.0 \%$ & \\
\hline & $>5$ & $15.7 \%$ & $51.7 \%$ & $32.6 \%$ & \\
\hline \multirow[t]{3}{*}{ Fashion Zone } & $<2$ & $14.8 \%$ & $63.0 \%$ & $22.2 \%$ & \multirow{3}{*}{$X^{2}=5.019, p=0.285$} \\
\hline & $2-5$ & $31.1 \%$ & $48.9 \%$ & $20.0 \%$ & \\
\hline & $>5$ & $15.7 \%$ & $60.7 \%$ & $23.6 \%$ & \\
\hline \multirow[t]{3}{*}{ His and Her } & $<2$ & $11.5 \%$ & $57.7 \%$ & $30.8 \%$ & \multirow{3}{*}{$X^{2}=0.512, p=0.972$} \\
\hline & $2-5$ & $11.1 \%$ & $60.0 \%$ & $28.9 \%$ & \\
\hline & $>5$ & $11.5 \%$ & $54.0 \%$ & $34.5 \%$ & \\
\hline \multirow[t]{3}{*}{ Health Zone } & $<2$ & $14.8 \%$ & $51.9 \%$ & $33.3 \%$ & \multirow{3}{*}{$X^{2}=10.115, p=0.039$} \\
\hline & $2-5$ & $22.7 \%$ & $56.8 \%$ & $20.5 \%$ & \\
\hline & $>5$ & $6.7 \%$ & $52.2 \%$ & $41.1 \%$ & \\
\hline \multirow[t]{3}{*}{ Café 48} & $<2$ & $3.7 \%$ & $40.7 \%$ & $55.6 \%$ & \multirow{3}{*}{$X^{2}=2.724, p=0.605$} \\
\hline & $2-5$ & $2.2 \%$ & $55.6 \%$ & $42.2 \%$ & \\
\hline & $>5$ & $1.2 \%$ & $44.9 \%$ & $53.9 \%$ & \\
\hline \multirow[t]{3}{*}{ Kiosk 48} & $<2$ & $0.0 \%$ & $40.7 \%$ & $59.3 \%$ & \multirow{3}{*}{$X^{2}=4.683, p=0.321$} \\
\hline & $2-5$ & $11.1 \%$ & $48.9 \%$ & $40.0 \%$ & \\
\hline & $>5$ & $9.0 \%$ & $40.4 \%$ & $50.6 \%$ & \\
\hline \multirow[t]{3}{*}{ Colleagues Concierge } & $<2$ & $11.5 \%$ & $50.0 \%$ & $38.5 \%$ & \multirow{3}{*}{$X^{2}=2.621, p=0.623$} \\
\hline & $2-5$ & $23.2 \%$ & $51.2 \%$ & $25.6 \%$ & \\
\hline & $>5$ & $16.3 \%$ & $55.8 \%$ & $27.9 \%$ & \\
\hline \multirow[t]{3}{*}{ Mind Zone } & $<2$ & $7.4 \%$ & $55.6 \%$ & $37.0 \%$ & \multirow{3}{*}{$X^{2}=4.948, p=0.293$} \\
\hline & $2-5$ & $20.5 \%$ & $50.0 \%$ & $29.5 \%$ & \\
\hline & $>5$ & $9.3 \%$ & $48.8 \%$ & $41.9 \%$ & \\
\hline \multirow[t]{3}{*}{ Dream Zone } & $<2$ & $48.0 \%$ & $36.0 \%$ & $16.0 \%$ & \multirow{3}{*}{$X^{2}=5.297, p=0.258$} \\
\hline & $2-5$ & $50.0 \%$ & $43.2 \%$ & $6.8 \%$ & \\
\hline & $>5$ & $35.4 \%$ & $44.3 \%$ & $20.3 \%$ & \\
\hline \multicolumn{6}{|l|}{ Relax Zone } \\
\hline \multirow[t]{3}{*}{ Game area } & $<2$ & $29.7 \%$ & $44.4 \%$ & $25.9 \%$ & \multirow{3}{*}{$X^{2}=0.132, p=0.998$} \\
\hline & $2-5$ & $28.9 \%$ & $46.7 \%$ & $24.4 \%$ & \\
\hline & $>5$ & $27.0 \%$ & $47.2 \%$ & $25.8 \%$ & \\
\hline \multirow[t]{3}{*}{ Reading corner } & $<2$ & $14.8 \%$ & $55.6 \%$ & $29.6 \%$ & \multirow{3}{*}{$X^{2}=0.665, p=0.956$} \\
\hline & $2-5$ & $17.8 \%$ & $57.8 \%$ & $24.4 \%$ & \\
\hline & $>5$ & $20.2 \%$ & $52.8 \%$ & $27.0 \%$ & \\
\hline \multirow[t]{3}{*}{ Internet corner } & $<2$ & $22.3 \%$ & $48.1 \%$ & $29.6 \%$ & \multirow{3}{*}{$X^{2}=7.923, p=0.244$} \\
\hline & $2-5$ & $18.2 \%$ & $56.8 \%$ & $25.0 \%$ & \\
\hline & $>5$ & $27.3 \%$ & $36.4 \%$ & $36.4 \%$ & \\
\hline
\end{tabular}

*: $\mathrm{p}<0.05$

Viewing work experience as a differentiating factor in regard to perception of the new facilities, only the Health Zone showed any significant difference. Many possible theories, from Herzberg's (1966) motivationhygiene theory to the psychological dimensions of emotional exhaustion of Lee and Shin (2005), may be advanced to explain the importance of this facility change. Finally, while examining the effect of work experience, it is 
interesting that the group with the most experience (greater than five years) was the most positive. Finally, the relationship between all facility changes and motivation is presented:

Table 5. Cross-tabulation in term of O-Zone facilities' impact on employee motivation

\begin{tabular}{|c|c|c|c|c|c|}
\hline $\begin{array}{c}\text { Opinion of } \\
\text { O-Zone facilities } \\
\end{array}$ & Impact & Negative & Positive & $\begin{array}{c}\text { Very } \\
\text { Positive } \\
\end{array}$ & $X^{2}$ and $p$-value \\
\hline Wall of Fame' & $\begin{array}{l}\text { No } \\
\text { Yes }\end{array}$ & $\begin{array}{r}33.3 \% \\
7.6 \% \\
\end{array}$ & $\begin{array}{l}50.0 \% \\
46.8 \% \\
\end{array}$ & $\begin{array}{l}16.7 \% \\
45.6 \% \\
\end{array}$ & $X^{2}=8.386, p=0.015^{*}$ \\
\hline Fashion Zone & $\begin{array}{l}\text { No } \\
\text { Yes }\end{array}$ & $\begin{array}{l}25.0 \% \\
20.3 \% \\
\end{array}$ & $\begin{array}{l}58.3 \% \\
54.4 \% \\
\end{array}$ & $\begin{array}{l}16.7 \% \\
25.3 \% \\
\end{array}$ & $X^{2}=0.694, p=0.875$ \\
\hline His and Her & $\begin{array}{l}\text { No } \\
\text { Yes }\end{array}$ & $\begin{array}{l}8.3 \% \\
8.1 \% \\
\end{array}$ & $\begin{array}{l}83.4 \% \\
52.5 \% \\
\end{array}$ & $\begin{array}{r}8.3 \% \\
38.5 \% \\
\end{array}$ & $X^{2}=4.844, p=0.304$ \\
\hline Health Zone & $\begin{array}{l}\text { No } \\
\text { Yes }\end{array}$ & $\begin{array}{l}16.7 \% \\
11.2 \% \\
\end{array}$ & $\begin{array}{l}58.3 \% \\
48.8 \% \\
\end{array}$ & $\begin{array}{l}25.0 \% \\
40.0 \% \\
\end{array}$ & $X^{2}=1.385, p=0.709$ \\
\hline Café 48 & $\begin{array}{l}\text { No } \\
\text { Yes }\end{array}$ & $\begin{array}{l}0.0 \% \\
1.3 \% \\
\end{array}$ & $\begin{array}{l}58.3 \% \\
31.2 \% \\
\end{array}$ & $\begin{array}{l}41.7 \% \\
67.5 \% \\
\end{array}$ & $X^{2}=3.436, p=0.179$ \\
\hline Kiosk 48 & $\begin{array}{l}\text { No } \\
\text { Yes }\end{array}$ & $\begin{array}{r}16.7 \% \\
3.8 \% \\
\end{array}$ & $\begin{array}{l}50.0 \% \\
35.4 \% \\
\end{array}$ & $\begin{array}{l}33.3 \% \\
60.8 \% \\
\end{array}$ & $X^{2}=6.849, p=0.077$ \\
\hline Colleagues Concierge & $\begin{array}{l}\text { No } \\
\text { Yes }\end{array}$ & $\begin{array}{l}27.3 \% \\
13.1 \% \\
\end{array}$ & $\begin{array}{l}63.6 \% \\
47.4 \% \\
\end{array}$ & $\begin{array}{r}9.1 \% \\
39.5 \% \\
\end{array}$ & $X^{2}=10.363, p=0.035^{*}$ \\
\hline Mind Zone & $\begin{array}{l}\text { No } \\
\text { Yes }\end{array}$ & $\begin{array}{r}36.3 \% \\
6.3 \% \\
\end{array}$ & $\begin{array}{l}27.3 \% \\
44.3 \% \\
\end{array}$ & $\begin{array}{l}36.4 \% \\
49.4 \% \\
\end{array}$ & $X^{2}=12.952, p=0.005^{*}$ \\
\hline Dream Zone & $\begin{array}{l}\text { No } \\
\text { Yes }\end{array}$ & $\begin{array}{l}45.5 \% \\
38.3 \% \\
\end{array}$ & $\begin{array}{l}54.5 \% \\
42.5 \% \\
\end{array}$ & $\begin{array}{r}0.0 \% \\
19.2 \% \\
\end{array}$ & $X^{2}=9.527, p=0.049 *$ \\
\hline Relax Zone & & & & & \\
\hline Game area & $\begin{array}{l}\text { No } \\
\text { Yes }\end{array}$ & $\begin{array}{l}45.4 \% \\
15.0 \% \\
\end{array}$ & $\begin{array}{l}45.5 \% \\
45.0 \% \\
\end{array}$ & $\begin{array}{r}9.1 \% \\
40.0 \% \\
\end{array}$ & $X^{2}=7.402, p=0.060$ \\
\hline Reading corner & $\begin{array}{l}\text { No } \\
\text { Yes }\end{array}$ & $\begin{array}{r}54.5 \% \\
8.7 \% \\
\end{array}$ & $\begin{array}{l}45.5 \% \\
53.8 \% \\
\end{array}$ & $\begin{array}{r}0.0 \% \\
37.5 \% \\
\end{array}$ & $X^{2}=18.481, p=0.000 *$ \\
\hline Internet corner & $\begin{array}{l}\mathrm{No} \\
\mathrm{Yes}\end{array}$ & $\begin{array}{l}45.5 \% \\
18.7 \%\end{array}$ & $\begin{array}{l}54.5 \% \\
42.5 \%\end{array}$ & $\begin{array}{r}0.0 \% \\
38.8 \%\end{array}$ & $X^{2}=9.026, p=0.029 *$ \\
\hline
\end{tabular}

$*: \mathrm{p}<0.05$

When respondents were asked directly to rank the facility changes in terms of their impact on motivation, all previously reported $\mathrm{O}$-Zone facility changes were named, thereby giving added support to our exploration of the relationship between facility perception and the independent factors of department and length of work. Additionally, the Wall of Fame and Dream Zone showed significant differences as well, possibly reflecting recent activities being held in these areas.

\section{CONCLUSIONS}

1. The O-Zone, while at its most basic level impressively improved Herzberg's hygiene conditions for employees at the Mandarin Oriental Bangkok, also significantly enhanced the recognition and appreciation of employees by management, which studies have shown to be one of the main factors that motivate good performance in a number of different contexts (Analoui, 2000; Lundin and Soderholm, 1995).

2. The study, by showing that diverse departments react differently to the upgrade in facilities, further supports research (Simons and Enz,1995) challenging the assumption that all changes in HRM affect all employees equally.

3. Some hospitality departments (e.g., Housekeeping) seem to display particularly strong sensitivity to changes in the working environment.

4. Similar to previous research (Maroudas et al., 2008), Mandarin Oriental employees showed strong interest in personal development as indicated by the high positive scores in such areas as Mind Zone, Reading Corner and Health Zone. This concern for personal and professional growth provides a valuable opportunity for HRM to establish meaningful training and development programs.

5. In addition, attention to these self-actualization needs (Maslow, 1954) of employees "can, to some extent, overcome concerns about future job security by facilitating 'subjective security”" (Maroudas et al., 2008). 
This study also supports HRM and hospitality research which examines the role that organizational commitment and job satisfaction play in influencing employees to remain in an organization and the importance of designing effective retention strategies (Deery and Iverson, 1996; Deery and Shaw, 1999; Ghiselli et al., 1979, 2001; Deery, 2008).

\section{LIMITATIONS}

The study was cross-sectional, undertaken shortly after the initial launch of O-Zone. What effect the facility changes will have on employees over time was not examined. Similarly, respondent bias is another weakness associated with taking the survey shortly after the O-Zone introduction. One example would be a possible halo extension, since any changes in employee working conditions could create positive perceptions - the Hawthorne effect (Landsberger, 1958). Another instance of bias could be the good-participant role, in which the participant attempts to discern the interviewer's hypotheses and to confirm them. The participant does not want to "ruin" the experiment (Nichols, A. L. and Maner, J. K., 2008).

\section{FUTURE RESEARCH}

Facilities, being more enduring than activities, may make more of a long-term impact on employee perception of motivation. This may hold implications for HRM; for example, the importance of permanent as opposed to temporary changes (such as training programs versus short-term shift rotation). Examination of the effect of activities on motivation should be examined in a separate study.

\section{COURSE RECOMMENDATIONS}

This case study is suitable for undergraduate and graduate level courses with emphasis on human resources, organizational behavior, and studies concerning motivation, working environment, and work-life balance.

\section{CASE STUDY QUESTIONS}

1. Critically evaluate employee perceptions of the O-Zone in terms of job rewards.

2. Explain the effects of the O-Zone on intrinsic motivation factors in employees. Provide examples when possible.

3. Explain the effects of the O-Zone on extrinsic motivation factors in employees. Provide examples when possible.

4. Discuss future challenges of the O-Zone in terms of employee expectations of the work environment.

\section{AUTHOR INFORMATION}

Dr. Thomas Marshall (contact author) is currently a full-time lecturer at Les Roches International School of Hotel Management, Les Roches Gruyère University of Applied Sciences, Switzerland. His research focus is on sponsorship, image match and destination marketing. thomas.marshall@lesroches.edu

Dr. Ewa M. Mottier is currently a full-time lecturer at Les Roches International School of Hotel Management, Les Roches-Gruyère University of Applied Sciences, Switzerland. Her research focuses on technology and employee sustainability management within the hospitality industry. ewa.mottier@lesroches.edu

Dr. Robert A. Lewis is currently a full-time lecturer at Les Roches International School of Hotel Management, Les Roches Gruyère University of Applied Sciences, Switzerland. His research focus is on flexible work methods and work-life balance. robert.lewis@lesroches.edu

\section{REFERENCES}

Analoui, F. (2000). What motivates senior managers? Journal of Managerial Psychology, 15(4), 324-340.

Deery, M. (2008). Talent management, work-life balance and retention strategies. International Journal of Contemporary 
Hospitality Management, 20(7), 792-806.

Deery, M. A. \& Iverson, R. D. (1996). Enhancing productivity: intervention strategies for employee turnover. In Johns, N. (Ed.), Productivity Management in Hospitality and Tourism (pp 68-95), London: Cassell.

Deery, M. \& Shaw, R. (1999). An investigation of the relationship between employee turnover and organizational culture. Journal of Hospitality and Tourism Research, 23(4), 387-400.

DiPietro, R. B. \& Condly, S. J. (2007). Employee turnover in the hospitality industry: An analysis based on the CANE model of motivation. Journal of Human Resources in Hospitality and Tourism, 6(1), 1-22.

Enz, C. \& Siguaw, J. (2000). Best practices in human resources. Cornell Hotel and Restaurant Administration Quarterly, 41(1), 48-61.

Ferrell, O. C. \& Weaver, K. M. (1978). Ethical beliefs of marketing managers. Journal of Marketing, 42(3), 69-73.

Ghiselli, R., La Lopa, J., \& Bai, B. (2001). Job satisfaction, life satisfaction and turnover intent among food-service managers. Cornell Hotel and Restaurant Administration Quarterly, 42(1), 28-37.

Herzberg, F. (1966). Work and the Nature of Man, Cleveland, OH: World Publishing Co.

Kantor, J. \& Weisberg, J. (2002). Ethical attitudes and ethical behavior: Are managers role models? International Journal of Manpower, 23(8), 687-703.

Kim, H. J., Tavitiyaman, P., \& Kim, W. G. (2009). The effect of management commitment to service on employee service behaviors: The mediating role of job satisfaction. Journal of Hospitality and Tourism Research, 33(369), 370390.

Landsberger, H. A. (1958). Hawthorne Revisited, Ithaca, NY: Cornell University.

Lee, K. E. \& Shin, K. H. (2005). Job burnout, engagement and turnover intention of dieticians and chefs at a contract foodservice management company. Journal of Community Nutrition, 7(2), 100-106.

Lewis, R. A. \& Mottier, E. M. (2011). A “hotel within a hotel” in Bangkok. Emerald Emerging Markets Case Studies Collection, 2(8), 1-9.

Lucas, R. (2002). Fragments of HRM in hospitality? Evidence from the 1998 workplace employee relation survey. International Journal of Contemporary Hospitality Management, 14(5), 207-212.

Lundin, R. A. \& Soderholm, A. (1995). A theory of temporary organizations. Scandinavian Journal of Management, 11(4), 437-455.

Maslow, A. (1954). Motivation and Personality. New York, NY: Harper.

Maroudas, L., Kyriakidou, O., \& Vacharis, A. (2008). Employees' motivation in the luxury hotel industry: The perceived effectiveness of human-resources practices. Managing Leisure, 13, 258-271.

Michel, J. W., Kavanagh, M. J., \& Tracey, J. B. (2013). Got support? The impact of supportive work practices on the perceptions, motivation, and behavior of customer-contact employees. Cornell Hospitality Quarterly, 54, 161173.

Ng, T. W. H. \& Sorensen, K. (2008). Toward a further understanding of the relationships between perceptions of support and work attitudes: A meta-analysis. Group \& Organization Management, 33, 243-68.

Nichols, A. L. \& Maner, J. K. (2008). The good subject effect: Investigating participant demand characteristics. Journal of General Psychology, 135, 151-165.

Pfeffer, J. (1994). Competitive Advantage Through People. Boston, MA: Harvard Business School Press.

Peluchette, J. \& K. A. Karl. 2005. Attitudes toward incorporating fun into the health care workplace. The Health Care Manager 24:268-75.

Poulston, J. M. (2009). Working conditions in hospitality: Employees' views of the dissatisfactory hygiene factors. Journal of Quality Assurance in Hospitality and Tourism, 10(1), 23-43.

Rowley, G. \& Purcell, K. (2001). 'As cooks go, she went': Is labour churn inevitable? International Journal of Hospitality Management, 20(2), 163-185.

Schneider, B. \& Bowen, D. E. (1993). The service organization: human resource management is crucial. Organizational Dynamics, 21(4), 39-52.

Simons, T. \& Enz, C.A. (1995). Motivating hotel employees. Cornell Hotel and Restaurant Administration Quarterly, 36(1), 20-27.

Spector, P. E., Zapf, D., Chen, P. Y., \& Frese, M. (2000). Why negative affectivity should not be controlled in job stress research: Don't throw out the baby with the bath water. Journal of Organizational Behavior, 21, 79-95.

Tews, M. J., Michel, J. W., \& Stafford, K. (2003). Does fun pay? The impact of workplace fun on employee turnover and performance. Cornell Hospitality Quarterly, 54(4), 370-382.

Tsaur, S. H. \& Lin, Y. C. (2004). Promoting service quality in tourist hotels: the role of HRM practices and service behavior. Tourism Management, 25, 471-481. 